\title{
Polymer liquids fracture like solids
}

\author{
Huang, Qian; Hassager, Ole
}

Published in:

Soft Matter

Link to article, DOI:

10.1039/c7sm00126f

Publication date:

2017

Document Version

Peer reviewed version

Link back to DTU Orbit

Citation (APA):

Huang, Q., \& Hassager, O. (2017). Polymer liquids fracture like solids. Soft Matter, 13, 3470-3474 . https://doi.org/10.1039/c7sm00126f

\section{General rights}

Copyright and moral rights for the publications made accessible in the public portal are retained by the authors and/or other copyright owners and it is a condition of accessing publications that users recognise and abide by the legal requirements associated with these rights.

- Users may download and print one copy of any publication from the public portal for the purpose of private study or research.

- You may not further distribute the material or use it for any profit-making activity or commercial gain

- You may freely distribute the URL identifying the publication in the public portal

If you believe that this document breaches copyright please contact us providing details, and we will remove access to the work immediately and investigate your claim. 


\title{
Polymer Liquids Fracture like Solids
}

\author{
Qian Huang and Ole Hassager \\ Department of Chemical and Biochemical Engineering, Technical University of Denmark, \\ DK2800 Kongens Lyngby, Denmark
}

\begin{abstract}
While fracture in brittle solids has been studied for centuries till today, there are few studies on fracture in polymer liquids. Recent developments in experimental techniques, especially the combination of controlled filament stretching rheometry and high speed imaging, have opened new windows into the detailed study of fracture processes for polymer liquids. High speed imaging shows that polymer liquids fracture like solids with initiation and propagation of an edge fracture. However, remarkable features such as highly reproducible critical stress, independent appearance of multiple fractures, and trumpet crack profiles, reveal mechanisms which are significantly different from solids.
\end{abstract}

Entangled polymer melts and solutions have been known for some time to fracture in a manner similar to solids when exposed to sufficiently high deformation rates. Fracture has been observed in extrusion processes ${ }^{1,2}$ and in the breakage of filaments $^{3,4}$. It has long been recognized that fracture is an abrupt process distinct from more smooth processes such as necking (ductile failure) and surface tension-driven breakup ${ }^{5}$. However the physics of the fracture process in itself still remains largely uncharted land. Part of the problem is the difficulty of subjecting a liquid to a precisely controlled deformation in the same way it is possible with solids. In this highlight we begin with reviewing experimental methods for extensional rheometry. We shall show that although polymer liquids are termed viscoelastic meaning both liquid-like and solid-like, the distinction between liquid and solid can be made very sharp. Thus in the stretching of entangled polymer solutions there exists a critical stretch rate dividing liquid and solid behavior. If stretched slower than this critical stretch rate, the solutions respond as liquids in the sense that steady flow is ultimately achieved with in principle arbitrarily large deformations. Conversely if the systems are stretched faster than this critical rate, they fracture like solids.

A seemingly straightforward extensional experiment ${ }^{6}$ to test if a material is a liquid is to hold a sample of initial length $L_{0}$ at the two ends and stretch it to length $L$. The overall deformation of the sample is then characterized by the nominal Hencky strain $\epsilon_{N}=\ln \left(L / L_{0}\right)$. The nominal Hencky strain rate defined as the time derivative $\dot{\epsilon}_{N}=d \epsilon_{N} / d t$ is kept constant in a start-up experiment. Alternatively one may utilize a pair of rotating cylinders ${ }^{7}$ to roll up a filament of the sample. In this situation the nominal Hencky strain rate is computed from the geometry and the constant rotational velocity of the cylinders. The nominal Hencky strain is then determined as $\epsilon_{N}=\dot{\epsilon}_{N} t$ where $t$ is the elapsed time. We focus here on flow driven by external motion not be surface tension of the filaments ${ }^{8,9}$. However even in the absence of surface tension filaments of viscoelastic liquids may undergo a necking instability leading to rupture. This has 
been in simulations ${ }^{10,11}$ and in analyses ${ }^{12}$ of viscoelastic liquid filaments subjected to a constant nominal Hencky strain rate. The necking instabilities ultimately lead to rupture of the filaments, which prevent steady flow. This process is a fluid mechanical phenomenon distinct from solid-like fracture. Malkin and coworkers ${ }^{4,13,14}$ proposed a master curve dividing the behavior of polymer liquids into four zones by plotting the nominal strain at rupture $\left(\epsilon_{c}\right)$ as a function of stretch rate (Fig.1a). The master curve shows that steady state flow can be observed at very slow rates in Zone I only, while necking instabilities take place at faster rates in Zones II and III. At very high rates in Zone IV, a glass-like behavior is observed and the materials fracture in a solid-like fashion.

\section{Controlled Filament Stretching Rheometry.}

Although the Malkin master curve has been reproduced in several experiments ${ }^{15-18}$, the recently developed controlled filament stretching has been able to achieve steady flow in Zones II and III also ${ }^{19-21}$. The method rests on the near incompressibility of soft materials, with the consequence that the local stretching of a filament may be obtained from measurements of the diameter. By local we mean any cylindrical slice that contains the same liquid at all times. Consider such a slice of initial diameter $D_{0}$ that is stretched so the diameter is reduced to $D(t)$ at time $t$. Then the true Hencky strain is defined by $\epsilon=-\frac{1}{2} \ln \frac{D(t)}{D_{0}}$ and the true Hencky strain rate is defined as $\dot{\epsilon}=\frac{d \epsilon}{d t}$. Controlled filament stretching rheometry (FSR, see e.g. VADER 1000, Rheo Filament $\mathrm{ApS}$,) allows for a start-up experiment in which a filament is stretched at constant stretch rate in the plane of minimum diameter with simultaneous measurements of the corresponding stress. The result of one such experiment is shown in Fig.1b which shows the axial stress $\left(\sigma_{z z}-\sigma_{r r}\right)$ as a function of Hencky strain at 5 different constant values of the Hencky strain rates. The liquid is a $33 \%$ solution of nearly monodisperse polystyrene in oligomeric styrene. The dynamics for stretching of the polystyrene molecules is characterized by a Rouse time of $\tau_{R}=165 \mathrm{~s}^{21}$. The stretch rates are then reported in terms of the non-dimensional Weissenberg number $W i_{R}=\tau_{R} \dot{\epsilon}$. It is seen, that for Weissenberg numbers equal to and less than 11.3 , a steady extensional flow with constant stress is ultimately obtained (the brown curves). Conversely for a Weissenberg number 32.4 the filament ruptures like a rubber band and the experiment ends abruptly. This gives a clear distinction between liquid behavior and solid (fracture) behavior. Thus, when true Hencky rates are utilized rather than nominal hencky rates, the four zones in the Malkin plot are reduced to just two: Liquid and solid. The critical stretch rate $\dot{\epsilon}_{c}$ that divides the liquid-like behavior from the solid-like behavior for the material in Fig.1b corresponds to a Weissenberg number between 11.3 and 32.4 . 

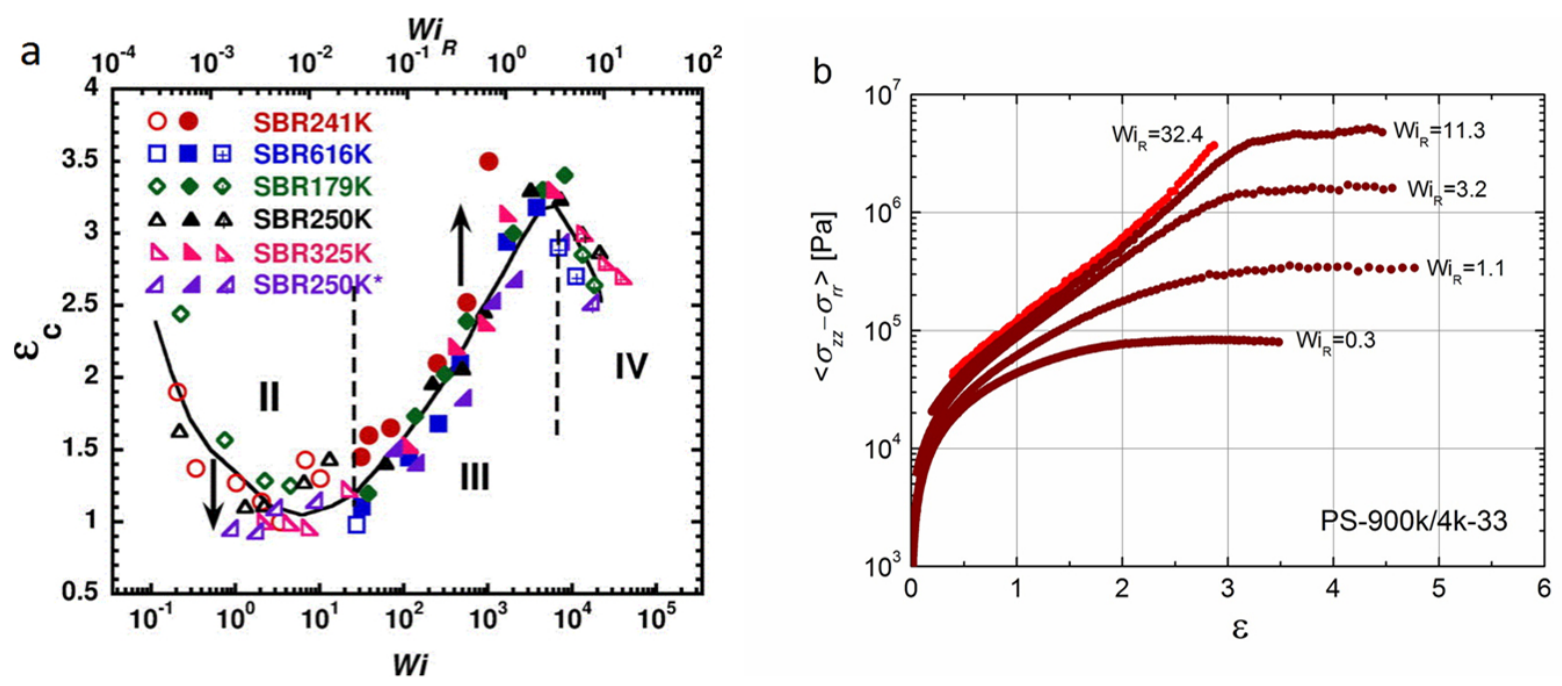

Figure 1: (a) Comparison of Malkin master curve with experimental data by plotting the nominal Hencky strain at rupture $\left(\epsilon_{c}\right)$ as a function of normalized stretch rate (Weissenberg number, Wi). Figure fromZhu and Wang ${ }^{18}$ with permission from the American Physical Society. (b) Measured extensional stress as a function true Hencky strain for a $33 \% 900 \mathrm{~kg} / \mathrm{mol}$ polystyrene solution at $130{ }^{\circ} \mathrm{C}$. Data reproduced from Huang et al. ${ }^{21}$.

The controlled FSR also gives the possibility of estimating the energy for fracture quantitatively, which allows for a further analysis of crack initiation and propagation in fracture process. The total work performed on a unit volume of the liquid when stretched to a given strain is in the form of an integral of the stress versus strain,

$$
E(\epsilon)=\int_{0}^{\epsilon}\left(\sigma_{z z}-\sigma_{r r}\right) d \epsilon^{\prime},
$$

where $\sigma_{z z}$ and $\sigma_{r r}$ are the axial and radial extra stress components respectively. When the material responds as a liquid $\left(\dot{\epsilon}<\dot{\epsilon}_{c}\right.$, the brown curves in Fig.1b), the integral increases continuously. Ultimately the work is dissipated into thermal energy. The steady extensional viscosity is obtained by dividing the stress by the strain rate. In this way, extensional viscosity functions have been obtained for a number of polymeric systems ${ }^{20-34}$. Specifically for a series of linear monodisperse polymers the steady stress has been shown to scale with the Rouse time based Weissenberg number ${ }^{19}$ suggesting that that the major contribution to stress comes from stretching and alignment of the polymer molecules in the flow direction. Even branched molecules behave as linear molecules in strong steady extensional flow ${ }^{35}$. Conversely when the material behaves as a solid $\left(\dot{\epsilon}>\dot{\epsilon}_{c}\right.$, the red curve in Fig.1b) the integral reaches a maximum value at the rupture strain. While there will be some dissipation in this situation, the stored elastic energy may still be estimated from Eq. (1) and a measurement of the elastic recovery.

High Speed Imaging. Most often the fracture process is too fast to observe without special aids. While necking instabilities have been shown to be an axisymmetric fluid mechanical instability ${ }^{36,37}$, recently the introduction of high speed imaging in soft material science ${ }^{38,39}$ has shown that fracture in polymer liquids is an edge fracture which breaks axisymmetry. Ligoure and co-workers from Montpellier studied a micro emulsion of oil droplets in water in which the droplets are linked by telechelic polymers. They let a (macroscopic) drop of the solution fall under gravity trailing behind a liquid filament as shown in Fig.2a. After about 71.50s, an edge fracture appears that rapidly ruptures the filament in about $3 \mathrm{~ms}$ as shown in Fig.2b. High speed 
imaging also allows analysis of the crack profile, which is parabolic for the micro emulsion as shown in Fig.2c. The appearance of a parabolic crack opening has all the signatures of an elastic fracture, so here is remarkable demonstration of solid behavior of a viscoelastic liquid.

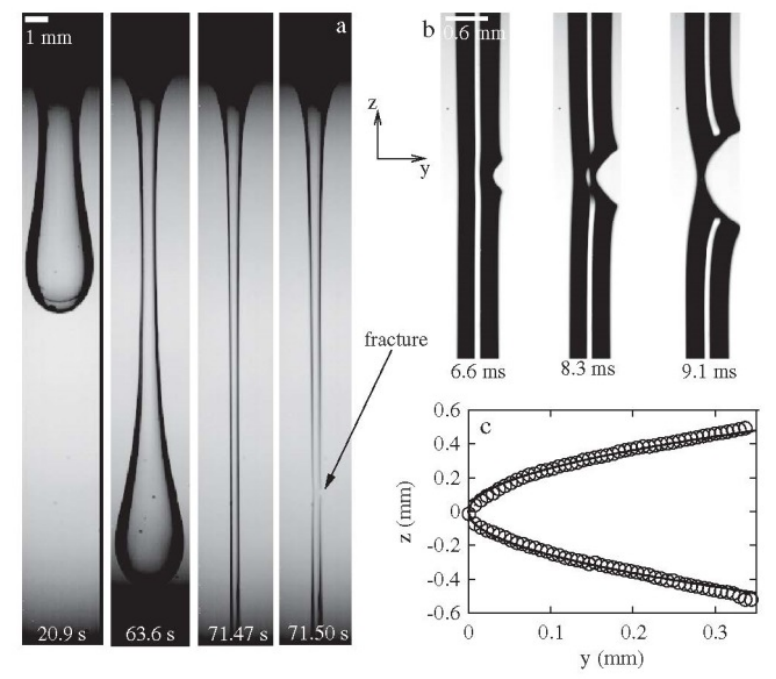

Figure 2: (a) Elongation and fracture of a drop of a solution of bridged micro emulsion under gravity. (b) Zoomed images of the fracture which propagates across the sample. (c) Typical parabolic fracture profile corresponding to the picture $\mathrm{b}$ (middle one) (circles). The black line represents a power law $z(y) \sim y^{1 / 2}$. Figure from Ref. ${ }^{38}$ with permission from the American Physical Society.

Fracture of Entangled Polymer Solution. Instead of the specially designed micro emulsion, Huang and coworkers ${ }^{40}$ studied more general polymer liquids in the form of entangled polymer solutions. They performed a sequence of experiments on two entangled polystyrene solutions with $864 \mathrm{~kg} / \mathrm{mol}$ diluted in $4 \mathrm{~kg} / \mathrm{mol}$ oligomeric styrene to $33 \%$ and $17 \%$ (denoted as PS-864k/4k-33 and PS-864k/4k-17 respectively). High speed imaging revealed that all ruptures occur via an edge fracture very similar to the fractures observed by Ligoure and coworkers. Moreover it was observed that the critical stress and strain for fracture are extremely reproducible functions of the stretch rate. In addition it was observed that once conditions for fracture have been reached, more than one crack is initiated and multiple cracks propagate simultaneously. Figure 3 shows the captured cracks in PS$864 k / 4 k-33$. The entire process of crack propagation is rather short (about 200ms) compared with the time for stretching (about 26s). Once the leading crack reaches the point of filament failure the stress is relieved and all other cracks close up upon removal of the driving force and only one fracture surface appears in the final state.

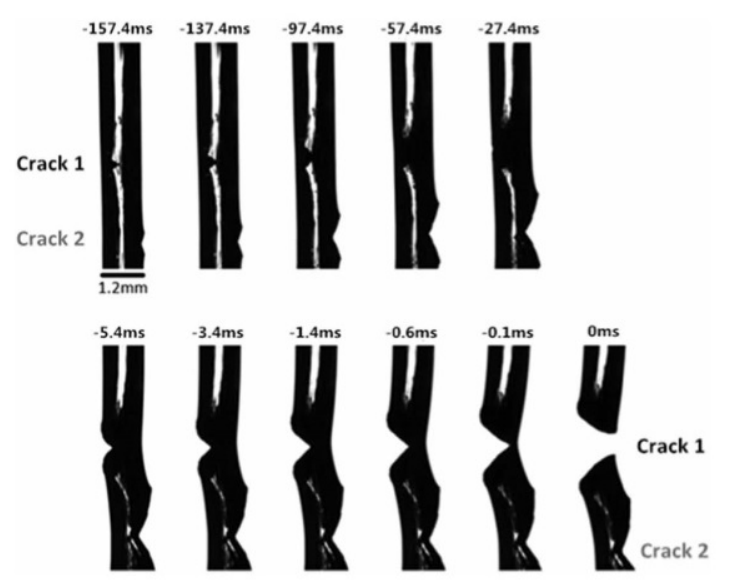

Figure 3: Crack propagation in uniaxial extensional flow for PS-864k/4k-33 stretched at $0.1 \mathrm{~s}^{-1}$ at $120^{\circ} \mathrm{C}$. Figure from Ref. ${ }^{40}$ with permission of the American Physical Society.

To underline the significance of the high reproducibility and the independent appearance of multiple fractures, we recall the basic aspects of fracture mechanics in brittle materials by Griffith $^{41}$. First of all to open a crack in a brittle material (by breaking of chemical bonds) will require an amount of energy per unit surface opened. In soft rubber materials this energy may be determined experimentally by a tearing experiment ${ }^{42}$, but it is less simple in liquids. Secondly, in the propagation of a crack in a filament under tension, this energy comes from 
relieving the stored elastic energy in the stretched filament. While the strain energy density may be estimated from the integral Eq.(1), it is only the energy in a small domain around the crack that contributes to the opening. In fact for a sufficiently small crack, the cost of creating new surface will always be larger than the energy relieved by the opening. In other words, to create a new crack in a completely homogeneous material will require jumping over an energy barrier. This has given rise to the idea that fracture in brittle materials depends on preexisting material imperfections in the form of micro cracks. The fracture propagates out from the largest pre-existing micro-crack also called the weakest link. This explains why fracture in brittle materials is seldom reproducible. But the high degree of reproducibility of the fractures observed by Huang and co-workers does not seem compatible with this explanation. Especially the simultaneous initiation and propagation of two cracks at different spatial positions is nearly impossible in a "weakest link" scenario ${ }^{43}$. As an alternative explanation Yves Pomeau ${ }^{44}$ suggested that thermal fluctuations may bring the system across the energy barrier needed for crack growth. This explanation explains both the reproducibility and the simultaneous appearance of multiple seemingly uncorrelated cracks. The thermally activated theory has also been invoked in connection with micro emulsions ${ }^{38,39}$ and ionomer melts ${ }^{45}$ as well.


Figure 4: (a) Fracture profile for entangled polymer solution. Figure from Huang et al. with permission of the American Physical Society. (b) Fracture profile for unentangled ionomer melt. Figure from Shabbir et al. ${ }^{45}$ with permission from the American Chemical Society. In both figures, $x$ represents distance from the fracture tip, while $u(x)$ is the fracture opening.

Crack Profile and Tip Conditions. The crack profile of PS-864k/4k-33 is shown in Fig.4a. The profile is described by the de Gennes viscoelastic trumpet model ${ }^{46}$. Close to the fracture tip the profile is described by $u(x) \sim x^{1 / 2}$ as in a glassy material. Further away (in most of the visible region) the profile is $u(x) \sim x^{3 / 2}$ as expected in a material with significant energy dissipation. It may seem counterintuitive, that energy dissipation should dominate anywhere in the crack zone in view of the high rates involved. However the rates away from the crack tip are in fact slow compared to the inverse relaxation time 
of a Kuhn segment. The inverse relaxation time of a Kuhn segment corresponds to the frequency where there is a transition from energy dissipation below the transition to energy storage in the glassy regime above the transition. Therefore the crack profile is in good agreement with the rheological characterization of the solution.

While the crack profile is well described it should be kept in mind that it is based on a stress analysis that must break down at the crack tip where a stress singularity is predicted. To understand the molecular mechanism at the crack tip, an analogy with brittle fracture in glassy polymers may prove useful. For glassy polymers it is generally recognized ${ }^{4748}$ that fracture is related to the phenomenon of crazing. Crazes are micron size planar defects that are spanned by an array of even smaller fibrils that carry the load. At the fracture stress the fibrils rupture and a crack is initiated and propagates. The rupture of the fibrils may be either by disentanglement or chain scission depending on molar mass, temperature and deformation rate $^{49}$. The question therefore is if the crack tip conditions for polymeric liquids involve disentanglement or chain scission or in fact both depending on the stretch rate. . Currently this is an unresolved question. Several investigators do propose that scission of the chemical bonds of the polymer chains between entanglements is involved. If so, That will bring the fracture of polymer liquids in line with the fracture of soft polymer networks ${ }^{50-52}$. Thus Malkin and co-workers ${ }^{53}$ suggest that at sufficiently high deformation rates, a tightening of the entanglement knots takes place. The result is that flow becomes impossible whereby the polymer chains rupture, as during the rupture of chemically crosslinked rubbers. Keep in mind, that the conditions for tightening of entanglement knots need only be present near the crack tip or if we accept the craze picture just in the fibrils. As mentioned, the measurements by Huang and coworkers $^{40}$ show that the deformation rates approach the inverse relaxation time of a single Kuhn segment and so it is not difficult to imagine, that the knots will indeed tighten up. Further support for chemical bond scission has been provided by S-Q Wang and co-workers ${ }^{17}$, who have used birefringence to show that the polymer chains are stretched out of the Gaussian regime at the conditions of rupture.

It is illustrative to contrast the fracture profiles for the entangled polymer melt with the profiles for an un-entangled ionomer melt ${ }^{45}$. As shown in Figure $4 b$, the fracture profile is parabolic indicating a fully elastic crack propagation process. The hypothesis here is that the cost of propagating the crack is associated not with chain rupture but with pullout of chains from the ionic clusters, a process that requires much less energy. Analysis of the crack profiles show that the deformation rates near the crack are in a regime where the elastic modulus dominates the viscous modulus in agreement with the parabolic crack opening. This parabolic profile is similar to the profile observed for the bridged micro emulsions $^{38,39}$ but is marked contrast to the profiles for entangled polymer systems.

In closing the following picture emerges for startup of extensional flow for entangled polymer solutions and melts: Below a critical strain rate, most entanglements are eventually lost and the system approaches steady flow. Above the critical strain rate, the entanglements tighten up and the filament fractures like a solid. The fracture differs markedly from the weakest link scenario in brittle solids in the appearance of a highly reproducible critical stress and independent appearance of multiple fractures. 
Acknowledgements:

Financial support from the Aage og Johanne Louis-Hansen Foundation is gratefully acknowledged. We would like to thank Professor A.Y. Malkin for constructive comments.

\section{References:}

1 J. H. Southern and D. R. Paul, Polym. Eng. Sci., 1974, 14, 560-566.

2 J. H. Southern, R. L. Ballman, J. A. Burroughs and D. R. Paul, J. Polym. Sci. Polym. Lett. Ed., 1978, 16, 157-160.

3 G. V. Vinogradov, Rheol. Acta, 1975, 14, 942-954.

4 A. Y. Malkin and G. V. Vinogradov, Polym. Sci. U.S.S.R., 1985, 27, 245-257.

5 Y. M. Joshi and M. M. Denn, J. Rheol. , 2003, 47, 291.

6 J. Matta and R. Tytus, J. Nonnewton. Fluid Mech., 1990, 35, 215-229.

7 M. L. Sentmanat, Rheol. Acta, 2004, 43, 657-669.

8 V. M. Entov and E. J. Hinch, J. Nonnewton. Fluid Mech., 1997, 72, 31-53.

9 A. V. Bazilevsky, V. M. Entov and A. N. Rozhkov, Fluid Dyn., 2011, 46, 613-622.

10 M. Yao, G. H. McKinley and B. Debbaut, J. Nonnewton. Fluid Mech., 1998, 79, 469501.

O. Hassager, M. I. Kolte and M. Renardy, J. Nonnewton. Fluid Mech., 1998, 76, 137151.

12 D. M. Hoyle and S. M. Fielding, J. Rheol. , 2016, 60, 1347-1376.

13 A. Y. Malkin, A. Arinstein and V. G. Kulichikhin, Prog. Polym. Sci., 2014, 39, 959-978.

1997, 41, 1-25.

15

V. C. Barroso and J. M. Maia, J. Nonnewton. Fluid Mech., 2005, 126, 93103.

\section{Y. Wang and S. Q. Wang, Rheol. Acta, 2010, 49, 1179-1185.}

Y. Wang and S. Q. Wang, Macromolecules, 2011, 44, 5427-5435.

X. Zhu and S.-Q. Wang, J. Rheol. , 2013, 57, 223-248.

A. Bach, K. Almdal, H. K. Rasmussen and O. Hassager, Macromolecules, 2003, 36, 5174-5179.

Q. Huang, O. Mednova, H. K. Rasmussen, N. J. Alvarez, A. L. Skov and O. Hassager, Macromelecules, 2013, 46, 5026-5035.

Q. Huang, L. Hengeller, N. J. Alvarez and O. Hassager, Macromolecules, 2015, 48, 4158-4163.

\section{G. H. Mckinley and T. Sridhar, Annu. Rev.} Fluid Mech., 2002, 375-415.

R. K. Gupta, D. A. Nguyen and T. Sridhar, Phys. Fluids, 2000, 12, 1296-1318.

P. K. Bhattacharjee, J. P. Oberhauser, G. H. McKinley, L. G. Leal and T. Sridhar, Macromolecules, 2002, 35, 10131-10148.

T. Sridhar, M. Acharya, D. A. Nguyen and P. K. Bhattacharjee, Macromolecules, 2014, 47, 379-386.

Q. Huang, M. Mangnus, N. J. Alvarez, R. Koopmans and O. Hassager, Rheol. Acta, 2016, 55, 343-350.

27 L. G. D. Hawke, Q. Huang, O. Hassager and D. J. Read, J. Rheol. , 2015, 59, 995-1017.

8 Q. Huang, S. Costanzo, C. Das and D. Vlassopoulos, J. Rheol. , 2017, 61, 35-47.

S. Costanzo, Q. Huang, G. lanniruberto, G. Marrucci, O. Hassager and D. 
Vlassopoulos, Macromolecules, 2016, 49, 3925-3935.

30 Q. Huang, S. Agostini, L. Hengeller, M. Shivokhin, N. J. Alvarez, L. R. Hutchings and O. Hassager, Macromolecules, 2016, 49, 6694-6699.

31 S. L. Wingstrand, N. J. Alvarez, Q. Huang and O. Hassager, Phys. Rev. Lett., 2015, $115,78302$.

D. M. Hoyle, Q. Huang, D. Auhl, D. Hassell, H. K. Rasmussen, A. L. Skov, O. G. Harlen, O. Hassager and T. C. B. McLeish, J. Rheol. , 2013, 57, 293.

N. J. Alvarez, J. M. R. Mar??n, Q. Huang, M. L. Michelsen and O. Hassager, Phys. Rev. Lett., 2013, 110, 168301.

34 Q. Huang, N. J. Alvarez, Y. Matsumiya, H. $\mathrm{K}$. Rasmussen, $\mathrm{H}$. Watanabe and $\mathrm{O}$. Hassager, ACS Macro Lett., 2013, 2, 741744.

G. Ianniruberto and G. Marrucci, Macromolecules, 2013, 46, 267-275.

S. M. Fielding, Phys. Rev. Lett., 2011, 107, 258301.

37 D. M. Hoyle and S. M. Fielding, Phys. Rev. Lett., 2015, 114, 158301.

H. Tabuteau, S. Mora, G. Porte, M. Abkarian and C. Ligoure, Phys. Rev. Lett., 2009, 102, 155501.

39 H. Tabuteau, S. Mora, M. Ciccotti, C.-Y. Hui and C. Ligoure, Soft Matter, 2011, 7, 9474-9483.

40 Q. Huang, N. J. Alvarez, A. Shabbir and O. Hassager, Phys. Rev. Lett., 2016, 117, 87801.
41 A. A. Griffith, Phil. Trans. R. Soc., 1921, A 221, 163-197.
42

43

44

45

46

47

48

49

50

51

52

53
G. J. Lake and A. G. Thomas, Proc. R. Soc. London. Ser. A, 1967, 300, 108-

J. Fineberg, Physics (College. Park. Md)., 2016, 9-10.

Y. Pomeau, C. R. Acad. Sci. Paris, 1992, 314, 553.

A. Shabbir, Q. Huang, Q. Chen, R. H. Colby, N. J. Alvarez and O. Hassager, Soft Matter, 2016, 12, 7606-7612.

P. G. de Gennes, Langmuir, 1996, 12, 4497-4500.

A. M. Donald and E. J. Kramer, Polymer (Guildf)., 1982, 23, 1183-1188.

A. M. Donald and E. J. Kramer, J. Polym. Sci. Part A-2, Polym. Phys., 1982, 20, 899909.

D. S. A. De Focatiis, C. P. Buckley and L. R. Hutchings, Macromolecules, 2008, 41, 4484-4491.

K. Mayumi, J. Guo, T. Narita, C. Y. Hui and C. Creton, Extrem. Mech. Lett., 2016, 6, 52-59.

1 A. Cristiano, A. Marcellan, B. J. Keestra, P. Steeman and C. Creton, J. Polym. Sci. Part B Polym. Phys., 2011, 49, 355-367.

2 C. Creton and M. Ciccotti, Reports Prog. Phys., 2016, 79, 46601.

A. Y. Malkin, A. V. Semakov and V. G. Kulichikhin, Polym. Sci. Ser. A, 2011, 53, 1198-1206. 


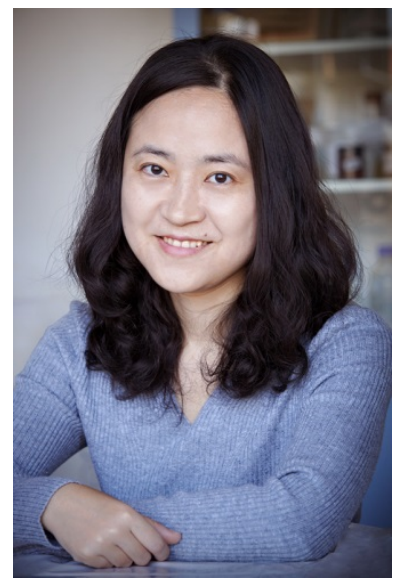

Qian Huang is currently a researcher in Chemical Engineering at Technical University of Denmark (DTU). She obtained her $\mathrm{PhD}$ degree at DTU in 2013 under the supervision of Prof. Ole Hassager. Her research focuses on extensional rheology and fracture behavior of polymer melts and solutions.

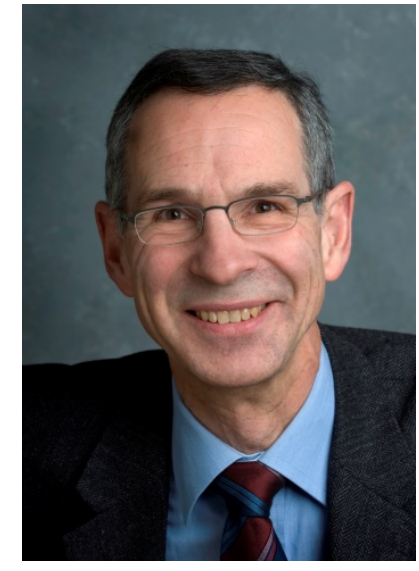

Ole Hassager is professor in Chemical Engineering at the Technical University of Denmark. His research interests lie in the areas of soft material physics, fluid mechanics, numerical simulation and rheology. He is coauthor of Dynamics of Polymeric Liquids, and co-founder of Rheofilament ApS. 